\title{
Dentists' views sought to improve oral cancer services
}

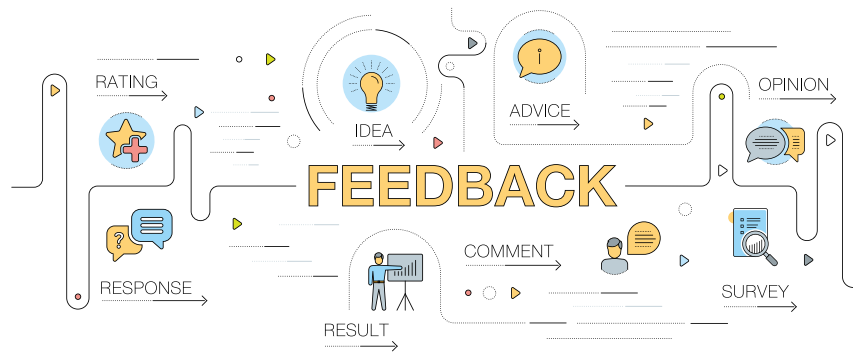

Dentists in Scotland are to be asked for their views on oral cancer services in a new survey being carried out by the BDA with the aim of improving prevention and earlier detection of the disease.

Oral cancer rates in Scotland are double those in England, and people from the most deprived communities are as much as three times more likely to be affected.

The BDA is surveying dentists north of the border to find out about their experiences of oral cancer services in their area.

Survey results will help shape an action plan and national referral pathway to be presented to ministers in the Scottish Government in November.

The intention is to improve care and outcomes for patients through prevention, early detection and uniform access to the best current surgical and restorative treatments.
The Oral cancer in Scotland - turning the tide survey ${ }^{1}$ asks about dentists' experience of:

- Preventative services such as smoking cessation

- Oral cancer services

- The potential impact of proposed changes to check-up intervals on detection.

Participants will also be asked for views on how to improve prevention and morbidity, as well as their experiences of dealing with patients with the condition.

The BDA said it had also jointly developed an oral cancer toolkit ${ }^{2}$ for dental health professionals with Cancer Research UK. The free toolkit was designed to help dental health professionals to identify and refer possible cases of oral cancer.

In the meantime, dentists and their teams had a vital role to play in ensuring oral cancers were detected early and patients were informed about the risk factors, said the union.

With oral cancers, early detection could result in a $90 \%$ survival rate, compared to a $50 \%$ survival rate for delayed diagnosis.

1. British Dental Association. Oral cancer in Scotland - turning the tide survey. Available at https://www.surveymonkey.co.uk/r/FJQGTDS (accessed on 10 September 2018).

2. BDA and Cancer Research UK. Oral Cancer Recognition Toolkit. Available at https:// www.doctors.net.uk/eClientopen/CRUK/oral_cancer_toolkit_2015_open/ (accessed on 10 September 2018. 